\title{
AGEs in Diabetes Prevention and Management
}

\author{
Youngwanichsetha $\mathbf{S}^{*}$ \\ Faculty of Nursing, Prince of Songkla University, Thailand
}

*Correspondence: Sununta Youngwanichsetha

Received on 31 December 2018; Accepted on 03 January 2019; Published on 07 January 2019

Copyright $(92019$ Youngwanichsetha S. This is an open access article and is distributed under the Creative Commons Attribution License, which permits unrestricted use, distribution, and reproduction in any medium, provided the original work is properly cited.

\section{Editorial}

Advanced glycation end products (AGEs) is known to be a risk factor associated with diabetes and its complications. AGEs are formed physiologically in all tissues and body fluids when carbonyl groups of reducing sugars react nonenzymatically with the free amino groups on proteins, Maillard reaction. Moreover, consumption of a diet high in AGEs is at risk for development of diabetes and chronic metabolic health problems including obesity, cardiovascular disease, renal disease, Alzheimer's, rheumatoid arthritis and others. This paper aimed to promote diabetes prevention and management by encouraging reduction on consumption of AGEs-rich diets and promoting consumption of antioxidants from natural foods [1].

Most of AGEs-rich diets are cooked foods and processed foods. Cooking methods with high temperature by grilling and deep frying also produce AGEs compound in foods. For example, 3 ounces of grilled chicken contains AGEs of $5,200 \mathrm{kU}, 1$ fried egg contains 1,240 kU. On the other hand, poached chicken contains AGEs of 1,000 kU, 1 scrambled egg contains AGEs only $75 \mathrm{kU}$. Therefore, unrecognizing of AGEs formation during cooking by heating sugars with red meat or fatty meat is prone to consumption of a high amount of AGEs [2].

In addition, usually, consumption of processed foods which is high in AGEs results in the development of obesity, insulin resistance, diabetes, and its complications. Most of the processed foods contain high sugar, salt, trans fat, and artificial substances. Trans fat includes margarine, mayonnaise, butter, and others. Example of processed food high in AGES, Hamburger contains AGEs of 5,418 kU [3].

After taking of diets containing AGEs, they can eliminate through kidney function. However, accumulation of AGEs in bloodstream and body tissues is long-lived damage. AGEs metabolism can generate intracellular free radical, oxidative stress and pro-inflammatory cytokines resulting in insulin resistance, endothelial cell damage, atherosclerosis, vasoconstriction. These pathogenesis conditions are associated with the development of diabetes and its complications. Therefore, reducing consumption of AGEs-rich diets is the concern [4].

Suggestions for overcoming AGEs pathogenesis in diabetes prevention and management are as follows:

- Health education of AGEs and its toxicity should be implemented to all.

- Choosing appropriate cooking methods such as steaming and boiling should be learned.

- Avoiding cooking meat with sugar by overheating at high temperature should be informed.

- Limiting the consumption of processed food should be promoted.

- Encouraging the consumption of natural foods rich in vitamin C, E and antioxidants from whole grain, legumes, fruit, and vegetables should be supported. 


\section{References}

1. Cavero-Redondo I, Soriano-Cano A, Alvarez-Bueno C, et al. Skin autofluorescene-indicated AGEs as predictors of cardiovascular and all-cause mortality in high-risk subjects: A systematic review and metaanalysis. J Am Heart Assoc. 2018;7(18):e009833.

2. Chen JH, Lin $\mathrm{X}, \mathrm{Bu} \mathrm{C}$, et al. Role of advanced glycation end products in mobility and considerations in possible dietary and nutritional intervention strategies. Nutr Metab (Lond). 2018;15:72.

3. Mirmiran P, Yousefi R, Mottaghi A, et al. Advanced glycation end products and risk of hypertension in Iranian adults: Tehran lipid and glucose study. J Res Med Sci. 2018;23:43.

4. Sharma C, Kaur A, Thind SS, et al. Advanced glycation end-products (AGEs): an emerging concern for processed food industries. J Food Sci Technol. 2015;52(12):7561-76. 